\title{
A Fourier-based method for the restoration of chopped and nodded images
}

\author{
M. Bertero ${ }^{1}$, P. Boccacci ${ }^{1}$, A. Custo ${ }^{1}$, C. De $\mathrm{Mol}^{2}$, and M. Robberto ${ }^{3, \star}$ \\ ${ }^{1}$ INFM and DISI, Università di Genova, via Dodecaneso 33, 16146 Genova, Italy \\ e-mail: boccacci@disi.unige.it; custo@disi.unige.it \\ 2 Département de Mathématique, Université Libre de Bruxelles, Campus Plaine CP217, 1050 Bruxelles, Belgium \\ e-mail: demol@ulb.ac.be \\ 3 Space Telescope Science Institute, 3700 San Martin Dr., Baltimore, MD 21218, USA \\ e-mail: robberto@stsci.edu
}

Received 28 November 2002 / Accepted 15 May 2003

\begin{abstract}
In a series of previous papers we have proposed and validated an iterative method, known as the projected Landweber method, for the restoration of astronomical images taken in chopping and nodding mode. While the method generally provides good results, it may also generate artifacts related to the huge non-uniqueness of the solution of the restoration problem. If the image satisfies additional boundary conditions, the non-uniqueness can be reduced, or even entirely removed. In this paper we investigate the case of periodic boundary conditions, which apply, in particular, to the case of a target area surrounded by a suitable region of empty sky. Periodic boundary conditions do not entirely remove the non-uniqueness of the solution, but allow using Fourier-based techniques. We introduce a new iterative method which can be considered as a relaxed and projected version of the van Cittert method. We formally demonstrate why this method does not produce the artifacts generated by the one we previously proposed, and we present numerical simulations confirming this result. We illustrate the convergence properties of the algorithm in the case of both compact and extended sources. Finally, we briefly discuss the potential and the limitations of the proposed technique.
\end{abstract}

Key words. techniques: image processing - methods: numerical

\section{Introduction}

In ground-based astronomical observations at mid-infrared wavelengths $(\lambda \simeq 5-20 \mu \mathrm{m})$ one faces the problem of extracting the weak astronomical signal from the overwhelming thermal background produced by the atmosphere and the telescope. The observing technique normally used is known as chopping and nodding. Chopping refers to the rapid modulation of the telescope beam between the target and an empty sky area. Nodding, or beam switching, refers to a second chopping sequence done with the telescope pointing to an offset position, so to have the target on the beam previously used to observe the empty sky (see, for instance, Allen 1975). If the target position is $\mathcal{A}$ and the two sky areas, at an angular distance $\pm \Delta$ (the chopping throw), are $\mathcal{B}$ and $\mathcal{C}$, then the final chopped and nodded signal is $I=-I(\mathcal{B})+2 I(\mathcal{A})-I(\mathcal{C})$. Thus, in the case of panoramic detectors, a chopped and nodded image is produced with each pixel containing twice the signal from the target minus the contributions from the two offset "sky" beams.

Send offprint requests to: $\mathrm{M}$. Bertero,

e-mail: bertero@disi.unige.it

* On assignment from the Space Telescope Division of the European Space Agency.
Depending on the amplitude of the chopping throw and on the size of the panoramic detector, the negative copies (counterparts) of the source may appear on the image shifted by $\pm \Delta$. Sources outside the main $\mathcal{A}$ field may also appear as spurious negative counterparts, if they fall within one of the offset beams. In general, a chopped and nodded image of a source more extended than the chopping throw is corrupted by the negative counterparts of the features located at a chopping throw distance. A restoration procedure is therefore required to recover the correct morphology and photometry of the target.

A first attempt to solve this problem was made by Beckers (1994). He proposed to use three images of the same target taken with optimized chopping amplitudes. Käufl (1995) applied this strategy, essentially an inverse Fourier filter without constraints, to simulated images and showed that it does not produce in general satisfactory results. Our team has taken a different approach, proposing an iterative method, the so-called projected Landweber method (Bertero et al. 1998), for approximating non-negative solutions of the restoration problem. The mathematical structure of the imaging matrix has been analysed in Bertero et al. (1999); the performance - and the 
limits - of the restoration algorithm have been demonstrated by Bertero et al. (2000) on real chopped and nodded images taken at the UKIRT telescope. In general, the method provides excellent results, especially if the data acquisition is carefully optimized, but it may also produce annoying artifacts. In Bertero et al. (1999) we have shown that the structure of the artifacts is somehow predictable, as it is related to the mathematical properties of the imaging matrix. In other words, artifacts are intrinsically related to the problem of extracting a particular solution from the huge set of all possible solutions, i.e. to the non-uniqueness of the solution of the restoration problem.

Special observing conditions can reduce the degree of nonuniqueness, and therefore the arbitrariness in the selection of a solution. In particular, it is possible to conceive the case where the offset fields $\mathcal{B}$ and $C$ contain only sources also visible in $\mathcal{A}$, i.e. there is empty sky at the upper and lower (relative to the chop/nod direction) field edges. Given the small format of current detector arrays and the sensitivity of large telescopes, this condition can hardly be satisfied by a single chopped and nodded image of a bright extended source. However, a mosaic of chopped and nodded images can be made large enough so that the extreme tiles satisfy this condition. A convenient observing technique therefore makes this case practically feasible. From a mathematical point of view, this is a particular case of periodic boundary conditions; besides decreasing substantially the nonuniqueness of the solution, the periodicity conditions allow a very simple mathematical treatment based on Fourier analysis and, for this reason, we consider this more general case. We investigate the intrinsic nature of the problem of reconstructing chopped and nodded images satisfying these additional conditions and we explore the potential and limitations of the corresponding restoration method.

\section{The mathematical model}

We denote by $f(\xi, \eta)$ the brightness distribution of the astronomical target, where $\xi, \eta$ are angular variables in the sky; this function is non-negative and can be assumed to vanish in regions of empty sky. The image of $f(\xi, \eta)$ obtained by means of the standard chopping and nodding technique will be denoted by $g(\xi, \eta)$. If chopping and nodding is done in the direction of the angular variable $\eta$, then the relationship between $g$ and $f$ is given by

$g(\xi, \eta)=-f(\xi, \eta-\Delta)+2 f(\xi, \eta)-f(\xi, \eta+\Delta)$,

where $\Delta$ is the chopping amplitude. The transfer function of the detection system is assumed to be uniform across the field and can then be set equal to one without affecting the results. An error term should be added to the right-hand side of this equation to account both for systematic and for random errors. Systematic errors (such as non uniform illumination, image distortion, PSF variations etc.) can be calibrated and possibly taken into account in the model of data acquisition; if not, they can produce residual artifacts as those discussed in Sect. 7. On the other hand, random errors (detector and photon noise, changes in the atmospheric transmission etc.) limit the accuracy of the restoration, as discussed in Sects. 6 and 7. For clarity, however, we introduce our basic formalism without explicitly indicating this error term.
Any image $g$ has a finite extent corresponding, in a first instance, to the field imaged by the panoramic detector; however, a mosaic of chopped and nodded images can cover a strip of sky, so that the domain $\mathcal{A}$ is in general a rectangle, i.e. $\mathcal{A}=\left[\xi_{\min }, \xi_{\max }\right] \times\left[\eta_{\min }, \eta_{\max }\right]$. As follows from Eq. (1), for a given value of $\xi$ in $\left[\xi_{\min }, \xi_{\max }\right], g$ receives contributions from the values of $f$ corresponding to values of $\eta$ in the interval $\left[-\Delta+\eta_{\min }, \eta_{\max }+\Delta\right]$, i.e. $f$ is defined on the domain $\mathcal{D}=\mathcal{A} \cup \mathcal{B} \cup \mathcal{C}$, namely the rectangle $\left[\xi_{\text {min }}, \xi_{\text {max }}\right] \times[-\Delta+$ $\left.\eta_{\min }, \eta_{\max }+\Delta\right]$.

The restoration problem consists in estimating the target $f$ from the knowledge of the detected image $g$; therefore, for each $\xi$, one has to solve a one-dimensional problem. If we neglect the dependence on $\xi$ of the function to be restored, the problem can be formulated as the solution of the following linear equation, obtained from Eq. (1):

$\left(D^{(2)} f\right)(\eta)=g(\eta)$

where $D^{(2)}$ is the second-difference operator defined by:

$\left(D^{(2)} f\right)(\eta)=-f(\eta-\Delta)+2 f(\eta)-f(\eta+\Delta)$.

Notice that the operator $D^{(2)}$ transforms a function defined on the interval $\left[-\Delta+\eta_{\min }, \eta_{\max }+\Delta\right]$ into one defined on the interval $\left[\eta_{\min }, \eta_{\max }\right]$.

Properties of $m$-th difference operators are investigated in Di Benedetto (2002), where a complete characterization of their null spaces and singular systems is given. The case $m=2$ corresponds to the operator $D^{(2)}$ defined in Eq. (3), the null space being then the set of the functions $f$ whose chopped and nodded image is identically zero. These functions can be called invisible objects and are responsible for the non-uniqueness of the solution of the restoration problem. The results proved in Di Benedetto (2002) imply that the set of the invisible objects is infinite dimensional so that the restoration problem is affected by a severe non-uniqueness. The singular system of the seconddifference operator, which provides an orthonormal basis in the set of the objects orthogonal to the invisible objects (namely those objects which are completely imaged with no loss of information), contains $2 q+1$ distinct singular values, where $q$ is the maximum integer contained in $\left(\eta_{\max }-\eta_{\min }\right) / \Delta$. All singular values have infinite multiplicity. In addition, the condition number, i.e. the ratio between the largest and the smallest singular value, is of the order of $(2 q / \pi)^{2}$ so that the problem is affected by a moderate ill-conditioning. We recall that the condition number controls the propagation of noise from the data to the solution. Indeed, the RMS relative error on the solution is bounded by the RMS relative error on the data multiplied by the condition number.

The non-uniqueness of the solution of the problem can be removed or at least reduced if the object $f(\eta)$ satisfies suitable boundary conditions. Conditions which lead to a very simple mathematical treatment of the problem are the periodic ones. As easily seen, it is possible to use such conditions if the object $f(\eta)$ is zero for all values of $\eta$ in the intervals $\left[-\Delta+\eta_{\min }, \eta_{\min }+\Delta\right]$ and $\left[-\Delta+\eta_{\max }, \eta_{\max }+\Delta\right]$. Indeed, in such a case, Eq. (1) holds true for the periodic extensions of $f(\eta)$ and $g(\eta)$, with period $D=\eta_{\max }-\eta_{\min }$. 
Let us then denote by $f_{l}$ the Fourier coefficients of $f(\eta)$, i.e.

$f_{l}=\frac{1}{D} \int_{0}^{D} f(\eta) \exp \left(-\mathrm{i} \frac{2 \pi}{D} l \eta\right) \mathrm{d} \eta, \quad l=0, \pm 1, \pm 2 \ldots$

and by $g_{l}$ those of $g(\eta)$. These coefficients become negligible for $|l|>l_{\max }$, as a consequence of the band-limiting of astronomical images. Then an easy computation shows that Eq. (2) implies the following equations for the Fourier coefficients:

$4 \sin ^{2}\left(\pi \frac{\Delta}{D} l\right) f_{l}=g_{l}$.

Notice that if $g_{0}$ is identically zero, then Eq. (5) is identically satisfied for $l=0$ and therefore $f_{0}$ is not determined by the equation. However, due to the presence of noise, it may happen that $g_{0}$ is not identically zero, so that Eq. (5) is not solvable for $l=0$. Knowing that this is an effect of the noise, one can always subtract $g_{0}$ from $g(\eta)$ and take $g(\eta)-g_{0}$ as the chopped and nodded image of $f(\eta)$. We can then conclude that the solution of the restoration problem is not unique because it is determined up to an arbitrary constant $f_{0}$.

\section{Discretization of the problem}

Let us now consider the discretization of the problem and denote by $\delta$ the angular size of the detector pixels and by $M$ and $N$ the numbers of rows and columns in the discrete image formed by the pixel values $g_{m, n}(m=0,1, \ldots, M-1 ; n=0,1, \ldots, N-1)$.

We assume, for simplicity, that the chopping amplitude $\Delta$ is an integer multiple $K$ of the pixel size $\delta$, i.e.

$\Delta=K \delta$.

In any case this conditions can be satisfied by a precise determination of the chopping-throw and a suitable rebinning of the image.

If we denote by $\xi_{0}, \eta_{0}$ the coordinates of the central point of a reference pixel $(0,0)$, then the coordinates of the central point of the pixel $(m, n)$ are given by

$\xi_{n}=\xi_{0}+\delta n ; \eta_{m}=\eta_{0}+\delta m$,

and the corresponding pixel value $g_{m, n}$ can be modeled as the integral of $g(\xi, \eta)$ over the pixel domain:

$g_{m, n}=\int_{-\delta / 2}^{\delta / 2} \mathrm{~d} \xi^{\prime} \int_{-\delta / 2}^{\delta / 2} \mathrm{~d} \eta^{\prime} g\left(\xi_{n}+\xi^{\prime}, \eta_{m}+\eta^{\prime}\right)$.

Here we assume that the pixel response is uniform over the pixel domain. If this condition is not satisfied, then the integral of $g$ must be replaced by a weighted integral of $g$, with a weight depending on the response function of the pixel.

Concerning the object $f$ to be imaged, we define its pixel values $f_{m, n}$ in the same way as the pixel values of $g$. The pixels of the region $\mathcal{A}$ correspond to the values of the indices $m, n$ indicated above; however, as already remarked, contributions to $g$ can come also from the regions $\mathcal{B}$ and $C$, so that the object must be represented on the complete region $\mathcal{D}=\mathcal{A} \cup \mathcal{B} \cup C$. The assumption made on $\Delta$ in Eq. (6) implies that $f_{m, n}$ must be defined also for the $m$-index values $-K,-K+1, \ldots,-1$ and $M, M+1, \ldots, M+K-1$ corresponding respectively to the pixel rows in $\mathcal{B}$ and $C$ which are not included in $\mathcal{A}$.
By inserting Eq. (1) and Eq. (6) into Eq. (8) and observing that $\eta_{m} \pm \Delta=\eta_{m \pm K}$, we see that the relationship between $g_{m, n}$ and $f_{m, n}$ is given by

$g_{m, n}=-f_{m-K, n}+2 f_{m, n}-f_{m+K, n}$.

Since the imaging process does not depend on $n$, the problem is $1 \mathrm{D}$ and the restoration of the object can be performed column by column. For this reason we will drop the index $n$; we will indicate as $\boldsymbol{g}$ a vector of length $M$ corresponding to a generic column of the array $g_{m, n}$ and as $f$ a vector of length $M+2 K$ corresponding to a generic column of the array $f_{m, n}$.

With this notation Eq. (9) can be written in the following form:

$A_{K} \boldsymbol{f}=\boldsymbol{g}$

where $A_{K}$ is a non-square matrix $M \times(M+2 K)$. It follows that the discrete version of the restoration problem defined in Eq. (2) corresponds to the solution of this linear equation and this is just the problem considered in our previous work.

On the other hand, if we assume that the functions $f$ and $g$ satisfy the periodicity condition, then, for each column, the vector $f$ of length $M+2 K$ coincides with the vector obtained from the periodic extension, of period $M$, of its components with $m=0,1, \ldots, M-1$. Therefore, if we denote now by $f$ the vector of length $M$ formed by the components with $m=0,1, \ldots, M-1$, it is possible to consider Eq. (9) as a relationship between the periodic extensions of $\boldsymbol{f}$ and $\boldsymbol{g}$. We can write this relationship in the following form:

$A_{C, K} \boldsymbol{f}=\boldsymbol{g}$,

where $A_{C, K}$ is the circulant $M \times M$ matrix given by:

$$
\begin{aligned}
\left(A_{C, K} \boldsymbol{f}\right)_{m}= & -f_{m+M-K}+2 f_{m}-f_{m+K}, \\
0 & \leq m<K \\
= & -f_{m-K}+2 f_{m}-f_{m+K}, \\
K & \leq m<M-K \\
& =-f_{m-K}+2 f_{m}-f_{m+K-M}, \\
& M-K \leq m \leq M-1 .
\end{aligned}
$$

The restoration problem can be defined again as the problem of solving this linear equation for each column of the image array.

\section{The imaging matrix}

The features of the restoration problem defined in Eq. (11) can be obtained by investigating the properties of the imaging matrix $A_{C, K}$. This matrix, as any circulant matrix, can be conveniently written in terms of a periodic point spread function (PSF):

$\left(A_{C, K}\right)_{m, m^{\prime}}=P_{m-m^{\prime}}^{(K)}$,

with $P_{m}^{(K)}$ given, in our case, by the periodic extension (with period $M$ ) of the vector

$P_{m}^{(K)}=2 \delta_{m, 0}-\delta_{m, K}-\delta_{m, M-K} \quad ; \quad 0 \leq m \leq M-1$,

where $\delta_{m, n}$ is the usual Kronecker symbol. 
The properties of $A_{C, K}$ can be derived from an analysis of its eigenvalues. It is well known (see, for instance, Bertero \& Boccacci (1998)) that the eigenvalues of a circulant matrix are given by the Discrete Fourier Transform (DFT) of the corresponding PSF. Therefore, by means of an elementary computation, we obtain from Eq. (14) that the eigenvalues of $A_{C, K}$ are given by

$\lambda_{l}^{(K)}=4 \sin ^{2}\left(\pi \frac{K}{M} l\right) ; l=0,1, \ldots, M-1$.

Since these eigenvalues are non-negative, it follows that the circulant matrix $A_{C, K}$ is symmetric and positive semi-definite; in addition it is singular because the eigenvalue $\lambda_{0}^{(K)}$ is always zero.

The dimension of the null space of the matrix $A_{C, K}$, as well as its condition number, depend on the value of the chopping amplitude $K$. By inspection of the eigenvalue spectrum of $A_{C, K}$, given in Eq. (15), it is easy to verify that the following proposition holds true:

Proposition 1. Let us assume that $M$ is a power of 2

$M=2^{p}$

and let us write the chopping amplitude $K$ in the following form

$K=2^{q} s$

where $s \geq 1$ is odd and $q$ is an integer, $0 \leq q<p$; then there exist $2^{q}$ eigenvalues $\lambda_{l}^{(K)}$ equal to zero, so that the dimension of the null space of $A_{C, K}$ is $2^{q}$; in addition there exist $2^{q}$ eigenvalues equal to 4 , the maximum eigenvalue of $A_{C, K}$.

In particular the null space of $A_{C, K}$ has dimension 1 when $K$ is odd $(q=0)$ while has dimension $K$ when $K$ is a power of $2(s=1)$. The case of $K$ odd is interesting because, in such an instance, the imaging matrix has very simple properties. Again by a simple inspection of the eigenvalue spectrum, it is easy to derive the following result:

Proposition 2. Let $M$ be as in Proposition 1 and let $K$ be odd. Then all the eigenvalues of $A_{C, K}$ have multiplicity 2, except 0 and 4 which have multiplicity 1; moreover they coincide with the eigenvalues of the matrix with $K=1$, even if the ordering depends on $K$. It follows that the condition number is independent of $K$ and given by

$\alpha^{(K)}=\frac{1}{\sin ^{2}\left(\frac{\pi}{M}\right)}=O\left(\frac{M}{\pi}\right)^{2}$,

so that it increases quadratically with the number of rows in the image.

Taking into account these results we now proceed to the problem of solving Eq. (11). By computing the DFT of both sides of this equation, we obtain

$4 \sin ^{2}\left(\pi \frac{K}{M} l\right) F_{l}=G_{l}$

where $\boldsymbol{F}$ and $\boldsymbol{G}$ are respectively the DFTs of $\boldsymbol{f}$ and $\boldsymbol{g}$. This is precisely a discrete version of Eq. (5) with the ratio $D / \Delta$ replaced by $M / K$. We point out that these two ratios coincide because the chopping amplitude satisfies the condition of Eq. (6).
Concerning the non-uniqueness of the solution, it is clear that, when $M$ is a power of 2 , the best choice corresponds to an odd value of $K$ because in such a case only the eigenvalue with $l=0$ is zero. Therefore, if $G_{0}=0$, Eq. (19) with $l=0$ is identically satisfied and the component $F_{0}$ is not determined by the data. On the other hand, if $G_{0}$ is not zero, this is an effect of the noise which can be removed by subtracting this constant from the measured $\boldsymbol{g}$. Such a procedure can be applied also in the general case of arbitrary values of $M$ and $K$, by zeroing all the components of $\boldsymbol{G}$ associated with zero eigenvalues.

This analysis allows us to conclude that, when $K$ is odd, all the Fourier components of $\boldsymbol{f}$ are uniquely determined except the one with $l=0$. In other words, $f$ is determined except for a constant. We also remark that the "smallest" solution, namely that with $F_{0}=0$, must be discarded because the sum of its pixel values is zero, so that it takes both positive and negative (large) values. Moreover, noise propagation from the data to the solution is controlled by the condition number given in Eq. (18), which can be large, so that the use of regularization methods is required (see Bertero \& Boccacci 1998). In conclusion, a viable approach to the problem requires the use of regularization methods with the additional constraint of non-negativity. A comment on this constraint, which has been proved to be essential in our previous work, may be appropriate at this point. Indeed, the restored non-negative solution will be affected by noise which is also non-negative. In particular, in a region of empty sky one gets a noise whose total flux is not zero. This may lead to problems in an astrophysical interpretation of the image but, as far as we know, there is no way for overcoming this difficulty while the advantages provided by non-negativity in the quality of the restoration are certainly considerable.

\section{The iterative restoration method}

The restoration problems considered above, and defined by Eq. (10) or Eq. (11), have non-negative solutions, and certainly more than one. Indeed, if we take any solution of these problems, containing both positive and negative values, and if we add to it a sufficiently large positive constant, we get another solution of the same problem which is also non-negative. As a consequence of the non-uniqueness of non-negative solutions, it is reasonable to look for that solution which has minimal norm (it is unique, because the set of the non-negative solutions is closed and convex) or, more precisely, for a regularized version of this solution since the restoration problem is ill-conditioned.

In our previous work (Bertero et al. 1998, 1999, 2000) we proposed the projected Landweber method (Eicke 1992; Bertero \& Boccacci 1998) as a viable approach to the regularized solution of the restoration problem of Eq. (10) with the additional non-negativity constraint. More precisely, the method is used to get the solutions of the least-squares equation:

$A_{K}^{T} A_{K} \boldsymbol{f}=A_{K}^{T} \boldsymbol{g}$,

where $A_{K}^{T}$ is the transposed of the matrix $A_{K}$. It also yields the solutions of Eq. (10) because this equation has exactly the same solutions of Eq. (20). For the reader's convenience we recall 
here the proposed iterative scheme:

$\boldsymbol{f}^{(0)}=0$,

$\boldsymbol{f}^{(k+1)}=P_{+}\left\{\boldsymbol{f}^{(k)}+\tau\left(A_{K}^{T} \boldsymbol{g}-A_{K}^{T} A_{K} \boldsymbol{f}^{(k)}\right)\right\}$,

where $k=0,1,2, \cdots \quad$ is the number of iterations, $P_{+}$is the projection operator onto the set of non-negative vectors (it sets to zero the negative components of the vector to which it is applied) and $\tau$ is a relaxation parameter satisfying the conditions

$0<\tau<\frac{2}{\lambda_{\max }}$,

$\lambda_{\text {max }}$ being the maximum eigenvalue of $A_{K}^{T} A_{K}$. In the following this method will be referred to as Method A.

This method can also be used in the case of periodic boundary conditions. However a modified approach is suggested by the remark that the matrix $A_{C, K}$ is square and positive semidefinite, so that the iterations can be applied directly to the linear equation of Eq. (11) instead of the least-squares equation of Eq. (20). The result is:

$\boldsymbol{f}^{(0)}=0$

$\boldsymbol{f}^{(k+1)}=P_{+}\left\{\boldsymbol{f}^{(k)}+\tau\left(\boldsymbol{g}-A_{C, K} \boldsymbol{f}^{(k)}\right)\right\}$,

with the same notations as in Eq. (21). The relaxation parameter $\tau$ must satisfy the conditions of Eq. (22), $\lambda_{\max }$ being now the maximum eigenvalue of $A_{C, K}$. Since we know that this maximum eigenvalue is 4 , we conclude that the relaxation parameter must be chosen in such a way that: $0<\tau<0.5$. Finally we observe that the iterative method defined above can be considered as the relaxed and projected version of a restoration method known as the van Cittert method (see, for instance, Frieden 1975; Bertero \& Boccacci 1998). In the following it will be called Method $B$.

The initialization of the iterations with $f^{(0)}=0$ is justified by the conjecture that, in such a way, one obtains an algorithm converging to the smallest (in the sense of the Euclidean norm) non-negative solution. We also point out that at the first iteration we have:

$\boldsymbol{f}^{(1)}=\tau P_{+} \boldsymbol{g}$,

so that initializing with $f^{(0)}=0$ is equivalent to initializing with a suitably scaled version of the detected image with the negative counterparts of the sources set to zero.

It must also be pointed out that Method B, as Method A, must not be pushed to convergence because the non-negative solutions of the imaging equations are strongly affected by noise propagation. As it is known (see Bertero \& Boccacci 1998), early stopping of the iterations has a regularizing effect similar to that obtained by introducing a smoothness constraint on the solution. Stopping criteria for Method A, which apply also to Method B, are discussed in Bertero et al. (2000).

A comparison of Method A and Method B shows that the main difference is that, in Method A, a "reblurring" of the residual $\boldsymbol{g}-A_{K} \boldsymbol{f}^{(k)}$ is performed by means of the matrix $A_{K}^{T}$. Such a reblurring, which is required because the matrix $A_{K}$ is nonsquare, introduces further positive and negative counterparts of the sources in addition to those already existing in $\boldsymbol{f}^{(k)}$; the final result is that it may produce the artifacts documented and discussed in Bertero et al. 2000. Since the reblurring is not required by Method $\mathrm{B}$, we expect to obtain restorations with much less artifacts. Obviously, both methods can be applied and compared in the case of images satisfying the periodic conditions.

In order to understand the properties of Method B, we briefly investigate the particular case of a "compact" source, namely a source which extends over a number of pixels smaller than the chopping amplitude $K$. If we assume that the image $g$ is not corrupted by noise and that the object $f$ satisfies this condition, then, for any $n$, the following exact relations hold true:

$\boldsymbol{g}=A_{C, K} \boldsymbol{f}, \quad P_{+} \boldsymbol{g}=2 \boldsymbol{f}$.

The first means that the data are noise-free, while the second one is a consequence of the non-superposition of the source and its negative counterparts. In such a case it is easy to prove the following result:

Proposition 3. If the object $\boldsymbol{f}$ and the image $\boldsymbol{g}$ satisfy the conditions of Eq. (25), then the $k$-th iterate of the algorithm of Eq. (23) is given by

$\boldsymbol{f}^{(k)}=\left\{1-(1-2 \tau)^{k}\right\} \boldsymbol{f}$.

The proof can be obtained by induction. Indeed the relation is true for $k=0,1$. We assume that it is true for $k$ and, in order to prove that it is also true for $k+1$, we replace Eq. (26) into Eq. (23); if we use the first relation of Eq. (25), we obtain:

$\boldsymbol{f}^{(k+1)}=P_{+}\left\{\left[1-(1-2 \tau)^{k}\right] \boldsymbol{f}+\tau(1-2 \tau)^{k} \boldsymbol{g}\right\}$.

Since negative values appear only in the second term at r.h.s. of this equation, while the first term is zero where the second one is negative (the source and its negative counterparts do not overlap), by taking into account the second relation of Eq. (25), we get:

$\boldsymbol{f}^{(k+1)}=\left[1-(1-2 \tau)^{k}\right] \boldsymbol{f}+2 \tau(1-2 \tau)^{k} \boldsymbol{f}$,

and the result is proved.

This result shows that the $k$ th iterate is a scaled version of the object $f$, with a scaling factor which tends to one when the number of iterations tends to infinity. Therefore all the iterates are completely free of artifacts. This is not true for Method A as a consequence of the re-blurring of the residual due to the matrix $A_{K}^{T}$. We illustrate this fact by means of a numerical example in the next Section.

Concerning convergence, Eq. (26) shows that it can be very fast because the constant multiplying $\boldsymbol{f}$ can converge very quickly to one (even if the rate of convergence may depend on the value of $\tau$ ). In general a few iterations are sufficient to reach an accuracy better than $1 \%$. However, as we show in the next Section by means of a numerical experiment, convergence can be slower in the case of complex objects and the restoration error may not tend to zero even in the absence of noise, although in practice it may become sufficiently small. 
We conclude this Section by observing that from the arguments used in the proof of Proposition 3 it follows that artifacts may appear in the restored images if we do not use the correct value of the chopping amplitude $K$. Indeed, if the image $\boldsymbol{g}$ satisfies the first relation of Eq. (25) but we use in the iterative algorithm of Eq. (23) a matrix $A_{C, K^{\prime}}$ corresponding to a different chopping amplitude $K^{\prime}$, then the negative counterparts of the source in $\boldsymbol{g}$ do not overlap exactly to the negative counterparts of the source in $A_{C, K^{\prime}} f$. As a consequence of this incomplete overlapping, the first iterations generate artifacts approximately spaced by $K$ with respect to the source. These artifacts propagate in the subsequent iterations, producing other weaker artifacts spaced by $2 K, 3 K$ etc., and therefore they look similar to the Type A artifacts produced by method A - see Bertero et al. (2000). However they are due now to the inexact knowledge of $K$ and are not intrinsic to the restoration method.

\section{Simulation study}

As for Method A (Bertero et al. 1999), the implementation of Method B is easy and fast, because the cyclic matrix $A_{C, K}$ has only five diagonals different from zero. For our simulation study, we compute at each iteration a relative restoration error defined as follows

$\rho^{(k)}=\frac{\left\|\boldsymbol{f}^{(k)}-\boldsymbol{f}\right\|_{2}}{\|\boldsymbol{f}\|_{2}}$

where $\|.\|_{2}$ denotes the Euclidean norm (square root of the sum of the squares of the pixel values), $\boldsymbol{f}^{(k)}$ is the result of the $k$ th iteration and $\boldsymbol{f}$ is the target object. This parameter allows us to verify that, in the presence of noise, both iterative methods have the semiconvergence property (see Bertero \& Boccacci 1998), i.e. the restoration error first decreases, reaches a minimum value and then increases monotonically. This property is related to the regularization effect of early stopping and, as a rule, in a simulation experiment the iterations must be stopped at the minimum of the restoration error.

The results derived in the previous Section in the case of compact sources are confirmed by numerical simulations both in the absence and in the presence of noise. Figure 1 shows a Gaussian shaped source, the corresponding chopped and nodded image obtained with $K=37$ (so that the object and its negative counterparts do not overlap), and the restorations obtained using Methods A and B. Concerning Method B, in the absence of noise the restoration error decreases up to a value corresponding to the machine approximation. The number of iterations required for obtaining a restoration error of the order of $0.1 \%$ depends on the relaxation parameter; for $2 \tau=0.9,0.7$, and 0.5 , we find that we need respectively 3,6 , and 10 iterations. In the presence of noise, the restoration error has the semiconvergent behaviour described above. If the chopped and nodded image is contaminated by white Gaussian noise with a standard deviation $\sigma$, the number of iterations required for reaching the minimum decreases with increasing noise (i.e. increasing $\sigma$ ). In general quite few iterations are required (less than 10), depending on the values of $\tau$ and $\sigma$; we also find that the most accurate estimates of the peak value and of the total number of counts of the object are obtained with a number of

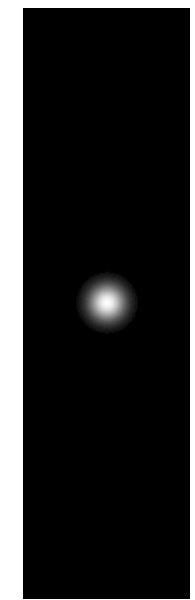

a)

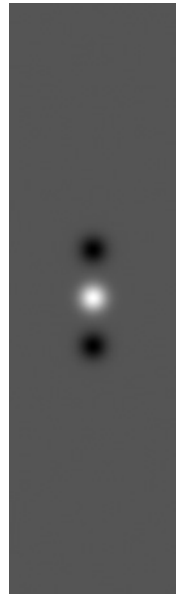

b)

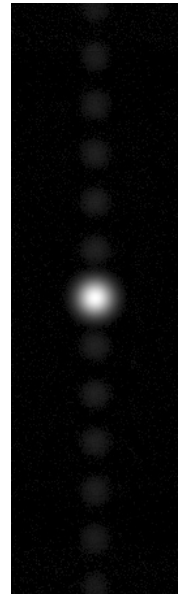

c)

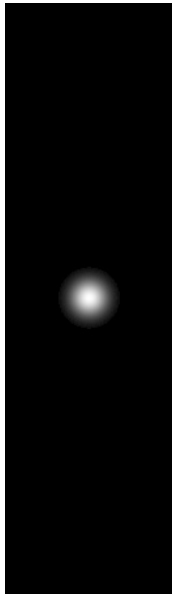

d)
Fig. 1. Restoration of a compact source: a) the object; b) the chopped $\&$ nodded image $(K=37)$; c) the restored image after 30 iterations of Method A; d) the restored image after 10 iterations of Method B. A logarithmic scale is used for displaying the object a) and the restored images c) and d) while a linear scale is used for displaying the chopped and nodded image $\mathbf{b}$ ).

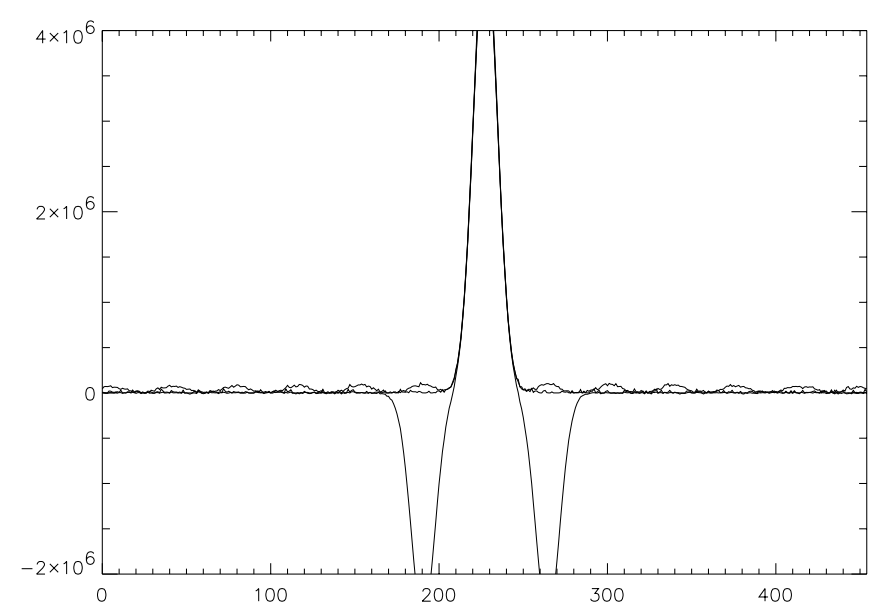

Fig. 2. Vertical cuts of the images of Fig. 1 through the points corresponding to the peak values. The smooth line with the negative counterparts is the cut of Fig. 1b (for comparison the profile has been divided by 2) while the two other lines correspond to Fig. 1c (that with the artifacts spaced by K) and to Fig. 1d.

iterations which is slightly larger than the number needed to reach the minimum of the restoration error.

The restorations obtained with Method B are free of artifacts, as discussed in the previous Section, while this is not true for Method A, as shown by Fig. 1c. In this experiment the image is contaminated by white Gaussian noise with $\sigma=10^{4}$ and the iterative algorithm is stopped at the minimum of the restoration error $(k=30)$. The typical artifacts of Method A, namely repeated images of the source (see Bertero et al. 2000) spaced by the chopping distance $\Delta$, are clearly visible in the restored image. The artifacts are also evident in Fig. 2 where we plot vertical cuts of the images of Fig. 1, taken through the points corresponding to the peak values. 


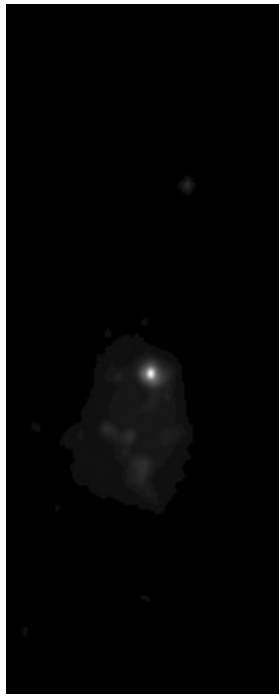

a)

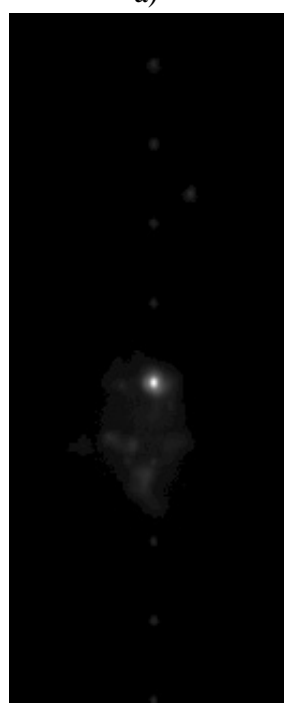

c)

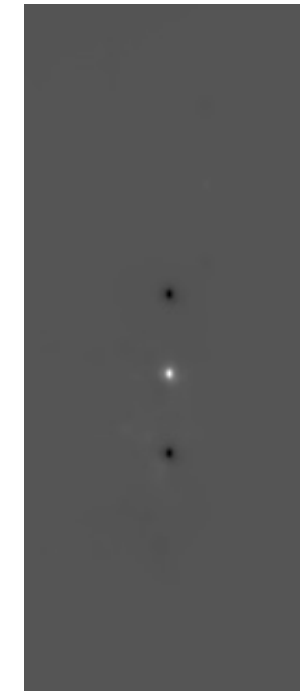

b)

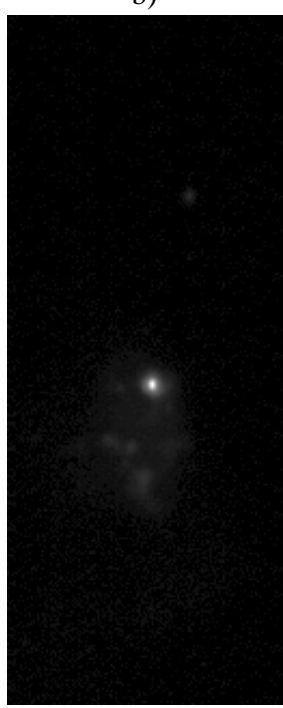

d)
Fig. 3. Comparison of the two methods in the restoration of an image of the $\mathrm{BN}$ system at $10 \mu \mathrm{m}$ : a) the original image; b) the corresponding chopped and nodded image with $K=37$; c) the restoration obtained with Method A; d) the restoration obtained with Method B.

In order to test the two methods in the case of an extended source, we use as a target $f$ a $10 \mu \mathrm{m}$ image of a region of the Orion Nebula centered on the Becklin-Neugebauer (BN) source. This image is part of a large image obtained at the $3.8 \mathrm{~m}$ United Kingdom Infrared Telescope (UKIRT) on Mauna Kea, Hawaii, using the Mid-IR camera MAX developed by the Max Planck Institut für Astronomie. The image has been obtained as a mosaic of chopped and nodded images restored by means of Method A. Artifacts in this case have been removed by combining images taken with different chopping throws and orientations (Robberto et al. 2003, in preparation). The field shown in Fig. 3a provides a severe test, as it contains complex structures with a very high dynamic range. From this field we obtain a (noise-free) chopped and nodded image corresponding to $K=37$. In addition, we consider a noisy version contaminated by a white Gaussian noise with zero mean

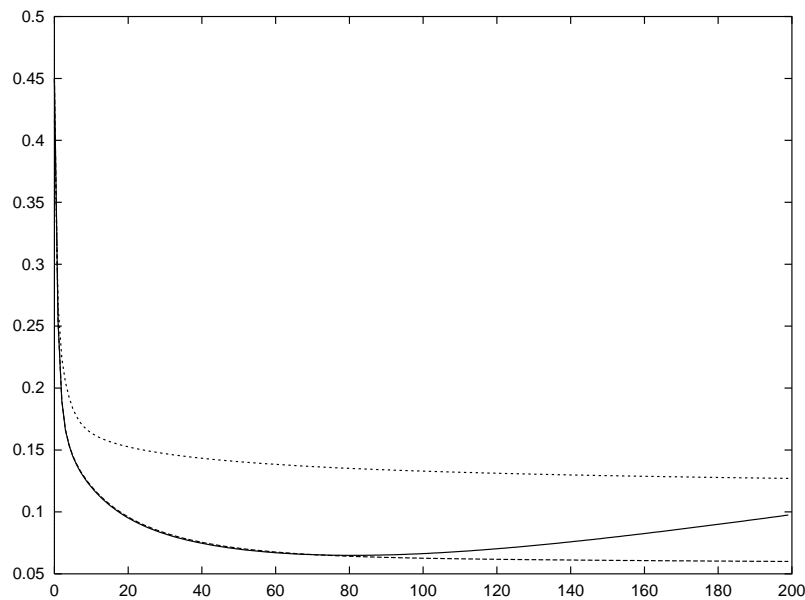

Fig. 4. Behaviour of the restoration error $\rho^{(k)}$ as a function of the number of iterations $k$ in the case of the numerical experiment based on the image of the BN system; Method A does not show a significant difference in the restorations of noise-free versus noisy data so that we plot only one error curve (dotted line); in the case of Method B, the dashed and the full line correspond, respectively, to noise-free and noisy data.

and standard deviation large enough to provide strong pixelization on top of the faintest structures. The difference between the cases with and without noise is negligible with respect to the effects of the artifacts, and therefore only the noisy version is presented in Fig. 3. In Fig. 3c we show the result obtained with Method A after 80 iterations while in Fig. 3d we show the result provided by Method B with the same number of iterations. Again, the restoration obtained with Method A is affected by artifacts, which clearly appear as replicas of the core of the BN system. The restoration provided by Method B does not contain these artifacts.

In Fig. 4 we plot the behaviour of the restoration error, defined in Eq. (29), as a function of the number of iterations $k$ in the case of the Orion/BN simulation. Since Method A does not provide significantly different errors in the two cases (noisefree and noisy data) we plot only the curve for the noisy data (dotted line). After a fast decrease at the very first iterations, the restoration error remains essentially stable with a value of about $15 \%$. Also for Method B there is no significant difference between the two cases until iteration 80; the error, however, is considerably smaller than with Method A (about 6\%). After iteration 80 the method shows in the noisy case the typical semiconvergent behaviour while providing an error which is essentially constant in the noise-free case. Such behaviour suggests that, in the case of complex objects, the approximation error relative to Method B does not tend to zero or, if it does, it tends to zero very slowly. Again, Method B provides a considerable reduction of the restoration error with respect to Method A (in this example by a factor 2.5 ). The relatively large number of iterations is related to the complexity of the source, whereas compact objects require much smaller number of iterations. 


\section{Concluding remarks}

In this paper we discuss an alternative to our previously proposed method for the restoration of chopped and nodded images. The new method is less general than the previous one since it can only be applied to the case where empty sky is observed above and below the target frame. Such a condition can be met no matter how extended is the source, by mosaicing a strip of adjacent images. The method provides results of higher quality than those obtained with the previous one. Our simulations show that the new method provides restorations essentially free from artifacts if the chopped and nodded images satisfy the boundary conditions and the chopping amplitude is known; we have shown that compensation of a fraction of pixel in the chopping amplitude can be introduced.

Nevertheless, a note of caution is needed. As any other image reconstruction method, the final results will depend on the quality of the original data. There are various pitfalls of data acquisition, especially in the mid-IR regime, that may limit the accuracy of the method. Optical field distortion can make the chopping throw variable across the image. The Point Spread Function may vary between the main and offset beams do to the seeing, or to the coma and astigmathism variations associated to the secondary tilting. Flat-field errors, changing atmospheric conditions and the natural variation of airmass affect the transfer function of the system, especially in the case of large mosaics. To some extent these problems, which are in general telescope and camera dependent, can be calibrated and corrected. Depending on the amount of residual effects, the restored image may be affected by artifacts, due this time not to the intrinsic nature of the problem or to the restoration method, but to errors in the image acquisition. We anticipate that these artifacts will appear as repeated and weaker versions of the bright sources (similar to the Type A artifacts defined in Bertero et al. 2000). Formally, they are due to the fact that the negative counterparts produced by $A_{C, K}$ in the iteration procedures do not match exactly to the negative counterparts of the sources in the original chopped and nodded image. Their intensity will depend on the amount of departure from the ideal conditions and on the brightness of the sources in the field. For best results it is therefore highly recommended to combine multiple observations with different chopping throws and, possibly, directions. In this case, our new method will provide extremely accurate reconstructions of the original field.

Acknowledgements. We thank an anonymous referee for helpful comments.

\section{References}

Allen, D. A. 1975, Infrared, the New Astronomy (New York: Halsted Press)

Beckers, J. M. 1994, Imaging with array detectors using chopping and other forms of differential detection, in Instrumentation in Astronomy VIII, ed. D. L. Crawf, \& E. R. Craine, Proc. SPIE, 2198, 1432

Bertero, M., \& Boccacci P. 1998, Introduction to Inverse Problems in Imaging (Bristol: IOP Press)

Bertero, M., Boccacci, P., \& Robberto, M. 1998, An inversion method for the restoration of chopped and nodded images, in Infrared Astronomical Instrumentation, ed. A. M. Fowler, Proc. SPIE, 3354,877

Bertero, M., Boccacci, P., Di Benedetto, F., \& Robberto, M. 1999, Restoration of chopped and nodded images in infrared astronomy, Inverse Problems, 15, 345

Bertero, M., Boccacci, P., \& Robberto, M. 2000, Wide-field imaging at mid-infrared wavelengths: Reconstruction of chopped and nodded data, PASP, 112, 1121

Di Benedetto, F. 2002, The $m$-th difference operator, Linear Algebra, to appear (the paper can be downloaded from http://www.dima.unige.it/ dibenede/ftp/preprints/ diff.ps)

Eicke, B. 1992, Iteration methods for convexly constrained ill-posed problems in Hilbert spaces, Num. Functional Anal. Opt., 13, 413

Frieden, B. R. 1975, Image enhancement and restoration, in Picture Processing and Digital Filtering, ed. T. S. Huang, 179 (Berlin: Springer-Verlag)

Käufl, H. U. 1995, Observing extended objects with chopping restrictions on $8 \mathrm{~m}$ class telescopes in the thermal infrared, in Calibrating and understanding HST and ESO instruments, ed. P. Benvenuti, ESO Conf. and Workshop Proc., 50, 159 